\title{
On the Role of Potentials in the Aharonov-Bohm Effect
}

\author{
Lev Vaidman \\ Raymond and Beverly Sackler School of Physics and Astronomy \\ Tel-Aviv University, Tel-Aviv 69978, Israel
}

\begin{abstract}
There is a consensus today that the the main lesson of the Aharonov-Bohm effect is that a picture of electromagnetism based on the local action of the field strengths is not possible in quantum mechanics. Contrary to this statement it is argued here that when the source of the electromagnetic potential is treated in the framework of quantum theory, the Aharonov-Bohm effect can be explained without the notion of potentials. It is explained by local action of the field of the electron on the source of the potential. The core of the Aharonov-Bohm effect is the same as the core of quantum entanglement: the quantum wave function describes all systems together.
\end{abstract}

PACS numbers: 03.65.-w, 03.65.Vf, 03.65.Ta, 03.65.Ud

Before the Aharonov-Bohm effect [1 (AB) was discovered, the general consensus was that particles can change their motion only due to fields at their locations, fields which were created by other particles. The main revolutionary aspect of the $\mathrm{AB}$ effect was that this is not generally true, and that in certain setups two particles, prepared in identical states, move in the same fields but end up in different final states. In particular, the electromagnetic field can vanish at every place where the electron has been, yet the electron motion is affected by the electromagnetic interaction. The $\mathrm{AB}$ effect states that the motion of an electron is completely defined by the potentials in the region of its motion and not just by the fields. The potentials depend on the choice of gauge, which cannot affect the motion of particles, but there are gauge invariant properties of the potentials (apart from the fields) that specify the motion of particles. The validity and the meaning of the $\mathrm{AB}$ effect has been extensively discussed [2 15]. I argue that there is an alternative to commonly accepted mechanism which leads to the effect, and that we might change our understanding of the nature of physical interactions back to that of the time before the $\mathrm{AB}$ effect was discovered. The quantum wave function changes due to local actions of fields.

The discussion will be on the level of gedanken experiments, without questioning the feasibility of such experiments in today's laboratory. Consider a Mach-Zehnder interferometer for electrons tuned in such a way that the electron always ends up in detector $B$, see Fig. 1 . We can change the electric potential in one arm of the interferometer such that there will be no electromagnetic field at the location of the wave packets of the electron but, nevertheless, the electron will change its behavior and sometimes (or it can be arranged that always) will end up in detector $A$. This is the electric AB effect. Alternatively, in the magnetic $\mathrm{AB}$ effect, the interference picture can be changed due to a solenoid inside the interferometer which produces no electromagnetic field at the arms of the interferometer.

Let us start our analysis with the electric $\mathrm{AB}$ effect. In the original proposal, the potential was created using conductors, capacitors etc. While those are closer to a practical realization of the experiment, a precise theoretical description of such devices is difficult. I consider, instead, two charged particles, the fields of which cancel at the location of the electron.

For simplicity of presentation, instead of the MachZehnder interferometer, I shall consider a one dimensional interferometer, see Fig. 2. (In fact, for an observer moving with a constant velocity in a perpendicular direction, this interferometer looks very much like the one described in Fig. 1.) The electron wave packet starts moving to the right toward a barrier which transmits and reflects equal weight wave packets toward mirrors $A$ and $B$. After reflection from the mirrors, the wave packets split again on the barrier. The interferometer is tuned in such a way that the there is a complete destructive interference toward mirror $A$, and the electron reaches mirror $B$ with certainty.

Another modification (the sole purpose of which is simplicity of the quantitative analysis of the experiment) is designing a special mirror for the electron which makes it spend a long time $\tau$ near it. For this purpose we intro-

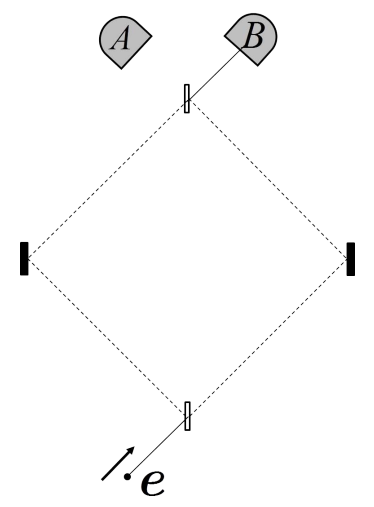

FIG. 1: Mach-Zehnder interferometer with electron as a test bed of the $\mathrm{AB}$ effect. Introducing relative electric potential between the arms of the interferometer or introducing a solenoid inside the interferometer spoils the destructive interference in detector $A$. 


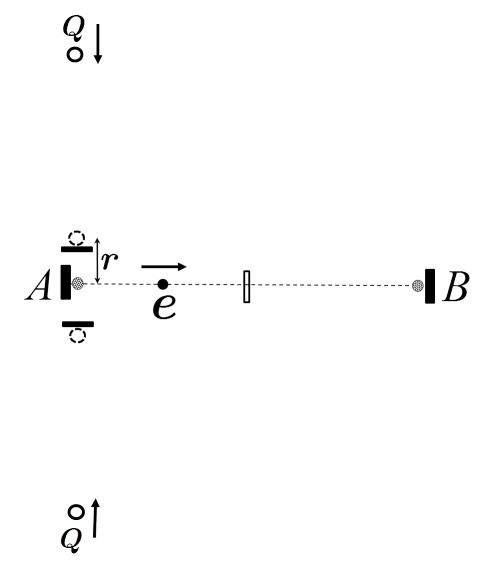

FIG. 2: A realization of the electric AB effect. Identical charges brought symmetrically to the electron wave packet in the left arm of the interferometer create potential for the electron without creating electric field at its location.

duce an interaction between the electron and the mirror with potential energy as a function of the electron distance from the mirror shown in Fig. 3. It goes to infinity at the surface of the mirror, smoothly becomes a constant value $V$ at $x \in(0, d)$, and smoothly goes to zero for $x>d$. The energy of the electron is only slightly higher than $V$. The dimensions of the interferometer are much larger than $d$ and we state that the electron is near the mirror when $x \in(0, d)$.

The source of the $\mathrm{AB}$ potential will be two particles of mass $M$ and charge $Q$ placed symmetrically on the perpendicular axis at equal large distances from mirror $A$. They have equal initial velocities toward the location of mirror $A$. At equal distance $r$ from the mirror, the charged particles bounce back due to other similarly designed mirrors, which make the charges spend a time $T$ near these mirrors. We choose $T<\tau$, so that the charges $Q$ are near their respective mirrors during the time the electron's wave packets are near their mirrors. We then can approximate the potential that the electron in the left arm feels as $\frac{-2 e Q}{r}$ for the time $T$. Indeed, when the

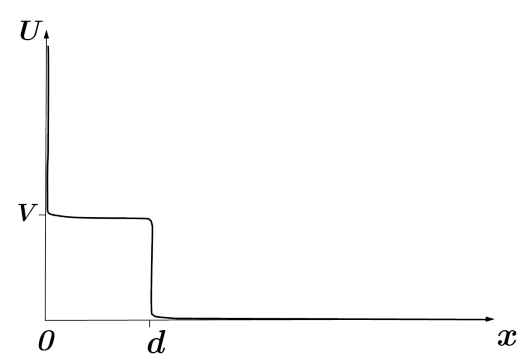

FIG. 3: The potential of the mirror forces. The potential energy of the particle as a function of its distance from the mirror. The particle with an energy slightly higher than $V$ spends long time near the mirror. charges are far away, their potential can be neglected, and the time the charges travel toward and from the mirror is much smaller than $T$. Thus, the phase difference between the two wave packets of the electron is:

$$
\phi_{A B}=\frac{-2 e Q T}{r \hbar} .
$$

The electron does not feel an electric field at any place where its wave packet passed, but it exhibits an interference pattern which is different from the pattern obtained in such an experiment by a neutral particle.

How can this result be understood if we consider all particles? The quantum state of the composite system is a superposition of two product states which I name branches. In the first one, the wave packet of the electron is on the left and in the other, it is on the right. The energy in the left branch is equal to the energy in the right branch, so energetic considerations cannot explain the phase difference. The electron does not experience any electric force, so the electron's wave packets are not shifted and thus cannot provide an explanation of the effect. The charges $Q$, however, do feel different forces in different branches. Thus, their wave packets in the left branch are slightly shifted relative to their wave packets in the right branch.

Let us calculate the shift of position of the wave packet of one of the two $Q$ charges due to its electromagnetic interaction with the electron. The shift is developed during the time $T$ when this charge is near its mirror. The interaction with the electron leads to a small perturbation in the motion of the charge and, since $d \ll r$, the velocity of the charge during this time, $v$, can be considered to be constant. The change in the kinetic energy of the charge due to its interaction with the electron allows us to find the change in its velocity and thus the shift $\delta x$ we are looking for:

$$
\frac{-e Q}{r}=\delta\left(\frac{M v^{2}}{2}\right) \simeq M v \delta v \quad \Rightarrow \quad \delta x=\frac{-e Q T}{M v r} .
$$

To observe the interference in the $\mathrm{AB}$ experiment, this shift should be much smaller than the position uncertainty of the charges. The de Broglie wavelength of the charge $\lambda=\frac{h}{M v}$. Both charges $Q$ are shifted in the same way creating the $\mathrm{AB}$ phase: $2 \frac{\delta x}{\lambda} 2 \pi=\phi_{A B}$.

The entanglement between the electron and the charges, which could have been created if the uncertainty in the velocity of the charges when they are near their mirrors is smaller than $\delta v$, disappears when the charges $Q$ travel back. Note however, that if, contrary to our assumption, the position uncertainty of the charges is smaller than $\delta x$, then the entanglement will remain and will lead to decoherence washing out the $\mathrm{AB}$ effect.

Let us turn now to the magnetic AB effect. I will show that the $\mathrm{AB}$ effect arises from different shifts of the wave packets of the source which feels different local electric 


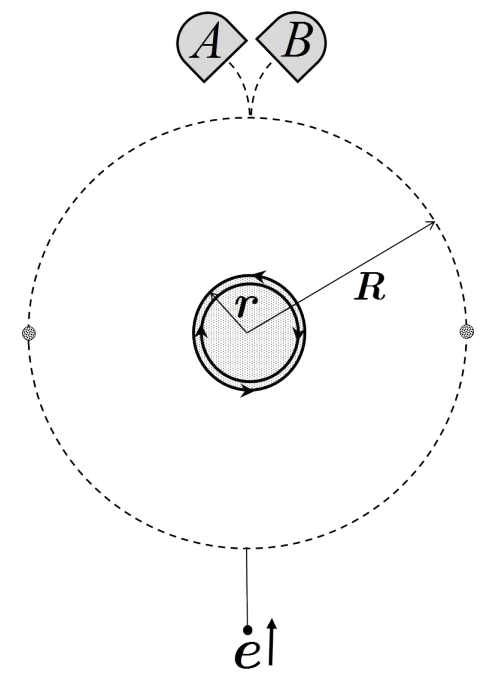

FIG. 4: The magnetic AB effect. The electron wave packet coming directly toward the solenoid splits into a superposition of two wave packets which encircle the solenoid from two sides and come out almost in the same direction, interfering toward detectors $A$ and $B$.

fields created by the left and the right wave packets of the electron.

Consider the following setup. The solenoid consists of two cylinders of radius $r$, mass $M$, large length $L$ and charges $Q$ and $-Q$ homogenously spread on their surfaces. The cylinders rotate in opposite directions with surface velocity $v$. The electron encircles the solenoid with velocity $u$ in superposition of being in the left and in the right sides of the circular trajectory of radius $R$, see Fig. 4.

The flux in the solenoid due to the two cylinders is:

$$
\Phi=2 \pi r^{2} \frac{4 \pi}{c} \frac{Q v}{2 \pi r L}=\frac{4 \pi Q v r}{c L} .
$$

Thus, the $\mathrm{AB}$ phase, i.e., the change in the relative phase between the left and the right wave packets due to the electromagnetic interaction is:

$$
\phi_{A B}=\frac{e \Phi}{c \hbar}=\frac{4 \pi e Q v r}{c^{2} L \hbar} .
$$

To simplify the alternative calculation based on direct action of the electromagnetic field, we assume $r \ll R \ll$ $L$. Before entering the circular trajectory, the electron moves toward the axis of the solenoid and thus it provides zero total flux through any cross section of the solenoid. During its motion on the circle, the magnetic flux through a cross section of the solenoid at distance $z$ from the perpendicular drawn from the electron is:

$$
\Phi(z)=\frac{\pi r^{2} e u R}{c\left(R^{2}+z^{2}\right)^{\frac{3}{2}}} .
$$

When the electron enters one arm of the circle, it changes the magnetic flux and causes an electromotive force on the charged solenoids which changes their angular velocity. In order to calculate the change in the velocity of the surface of the cylinder we have to integrate the impulse exerted on all thin slices of the cylinder. For simplicity, I consider here the surface motion as a linear motion. The contribution of a slice with an infinitesimal charge $d Q$ to the impulse is $\frac{\Phi(z) d Q}{c 2 \pi r}$ and integration over the slices yields:

$$
\delta v=\frac{1}{M} \int_{-\frac{L}{2}}^{\frac{L}{2}} \frac{\pi r^{2} e u R}{c^{2}\left(R^{2}+z^{2}\right)^{\frac{3}{2}}} \frac{1}{2 \pi r} \frac{Q}{L} d z \simeq \frac{u Q e r}{c^{2} M R L} .
$$

Then, the shift of the wave packet of a cylinder during the motion of the electron is:

$$
\delta x=\delta v \frac{\pi R}{u}=\frac{\pi Q e r}{c^{2} M L} .
$$

The relevant wavelength of de Broglie wave of each cylinder is $\lambda=\frac{h}{M v}$. For calculating the AB phase we should take into account that both cylinders are shifted and that they are shifted (in opposite directions) in both branches. This leads to a factor 4 and provides the correct expression for the AB phase: $4 \frac{\delta x}{\lambda} 2 \pi=\phi_{A B}$.

If the uncertainty in the velocity of the cylinders is smaller than $\delta v$, then, during the electron circular motion, the electron and the cylinders become entangled. But when the electron leaves the circular trajectory, it exerts an opposite impulse on the cylinders and this entanglement disappears.

I have explained both electric and magnetic $\mathrm{AB}$ effects through actions of local fields on the quantum wave function. The electron in states $|L\rangle_{e}$ and $|R\rangle_{e}$ causes, via action of its electromagnetic field, different evolutions for the quantum state of the source: $\left|\Psi_{L}\right\rangle_{S}$ and $\left|\Psi_{R}\right\rangle_{S}$. The total wave function of the electron and the source is

$$
\frac{1}{\sqrt{2}}\left(|L\rangle_{e}\left|\Psi_{L}\right\rangle_{S}+|R\rangle_{e}\left|\Psi_{R}\right\rangle_{S}\right) .
$$

During the evolution, the source states $\left|\Psi_{L}\right\rangle_{S}$ and $\left|\Psi_{R}\right\rangle_{S}$ might become orthogonal, or mostly differ only in their phase, but at the end of the process, the states of the source are identical except for the AB phase. Thus, the total wave function becomes

$$
\frac{1}{\sqrt{2}}|\Psi\rangle_{S}\left(|L\rangle_{e}+e^{i \phi_{A B}}|R\rangle_{e}\right),
$$

and the $\mathrm{AB}$ phase can be observed in the electron interference experiment.

The celebrated manifestation of a quantum wave function for a combined system is the nonlocal correlations which are generated by entangled states. The AB effect is conceptually different, since it can appear even if in the state (8) there is almost no entanglement at all times.

One might wonder why, instead of performing exact calculations in the framework of quantum mechanics, I consider particles and cylinders pushed by fields in the 
framework of classical mechanics and then use the correspondence principle to calculate the shifts of the quantum wave packets of particles and cylinders. I have to follow this path because the standard formulation of quantum mechanics and the Schrödinger equation in particular, are based on potentials. I hope that a general formalism of quantum mechanics based on local fields will be developed. It will provide a solution to the problem of motion of a quantum particle in a force field even if there is no potential from which it can be derived. Meanwhile my assertion provides one useful corollary: If the fields vanish at locations of all particles then these fields yield no observable effect.

Let us test this corollary. Consider a modification of the electric AB effect described above in which the charges $Q$ do not automatically perform their motion toward mirror $A$ and back, but only when the electron on the path $A$ triggers this motion, i.e., only in the left branch. I choose a particular value of the charge of the external particles, $Q=4 e$ for which the total electric field at the location of each particle created by other particles is zero. Neither the electron, nor the charges $Q$ feel an electromagnetic field in any of the branches. My assertion is that there will be no $\mathrm{AB}$ effect in this setup, in spite of the fact that the electron of the left branch has an electric potential, while the electron of the right branch has not. The original treatment of the $\mathrm{AB}$ effect is invalid since we do not have here a motion of an electron in a classical electromagnetic field, but a treatment of the problem using "private potential" created by induced charges 16] shows that indeed there is no AB effect in this case.

I believe that we can find an explanation of the kind presented above for any model of the AB experiment. However, the pictorial explanation of the creation of relative phase due to spatial shifts of wave packets disappears when we go beyond the physics of moving charges. We can replace the charged cylinders by a line of polarized neutrons producing magnetic flux due to quantum spins. In this case there is no spatial shift of wave packets. I am not aware of any pictorial explanation of the change of the phase of the spin state of the neutron, but contrary to the phase of the electron in the standard approach to the $\mathrm{AB}$ effect, the phases of neutrons are changed locally due to the magnetic field of the electron. This is also an explanation of the Aharonov-Casher (AC) effect [17]: the local electric field acting on the moving neutron is responsible for appearance of the AC phase. Note, however, that it does not lead to a classical lag of the center of mass of the neutron [18, 19.

I have not presented a general proof that in order to have an observable effect, the particles must pass through regions of nonzero fields. Rather, what I have shown is that the setups of electric and magnetic $\mathrm{AB}$ effects do not contradict this assertion. Note, however, that the last example in which there is an electric field almost everywhere except the locations of the particles and this field causes no effect, strongly supports my claim.

Since the electromagnetic potential at any point along the trajectory of the electron can be gauged away, the standard approach to the AB effect leads to a paradoxical, in my view, nonlocal feature of quantum mechanics: the AB phase which has observable manifestation is acquired inside the interferometer in spite of the fact that there is no particular place or time where this happens. I have shown that this peculiarity disappears when all relevant parts of the system are considered: the phase is gradually acquired by the source of the electromagnetic potential.

This result does not question the validity of the $\mathrm{AB}$ effect and does not diminish the importance of its numerous applications. It removes, however, conceptual claims associated with the AB effect regarding non-locality and the meaning of potentials. The AB effect does not prove that the evolution of a composite system of charged particles cannot be described completely by fields at locations of all particles. The potentials might be just a useful auxiliary mathematical tool after all.

I thank Noam Erez, Yaron Kedem, Shmuel Nussinov and Philip Pearle for useful discussions. This work has been supported in part by the Binational Science Foundation Grant No. 32/08 and the Israel Science Foundation Grant No. 1125/10,

[1] Y. Aharonov and D. Bohm, Phys. Rev. 115, 485 (1959).

[2] W.H. Furry and N.F. Ramsey, Phys. Rev. 118, 623 (1960).

[3] M. Peshkin, I. Talmi, and L.J. Tassie, Ann. Phys. 12, 426 (1961).

[4] Y. Aharonov and D. Bohm, Phys. Rev. 123, 1511 (1961).

[5] B. Liebowitz, Nuovo Cimento 38, 932, (1965).

[6] T.H. Boyer, Phys. Rev. D 8, 1679 (1973).

[7] T.T. Wu and C.N. Yang, Phys. Rev. D 12, 3845 (1975).

[8] P. Bocchieri and A. Loinger, Nuovo Cimento 47, 475 (1978).

[9] S.M. Roy, Phys. Rev. Lett. 44, 111 (1980).

[10] M. Peshkin, Phys. Rep. 80, 375 (1981).

[11] D.M. Greenberger, Phys. Rev. D 23, 1460 (1981).

[12] S. Olariu and I.I. Popescu, Rev. Mod. Phys. 57, 339 (1985).

[13] M. Peshkin and A. Tonomura, The Aharonov-Bohm effect. Springer-Verlag (1989).

[14] Y. Aharonov, T. Kaufherr, and S. Nussiov, J. Phys.: Conf. Ser. 173, 012020 (2009).

[15] A. Walstad, Int. J. Theor. Phys. 49, 2929 (2010).

[16] T. Kaufherr, Y. Aharonov, S. Nussinov, S. Popescu and J. Tollaksen, Phys. Rev. A 83, 052127 (2011).

[17] Y. Aharonov and A. Casher, Phys. Rev. Let. 53, 319 (1984).

[18] T.H. Boyer, Phys. Rev. A 36, 5083 (1987).

[19] Y. Aharonov, P. Pearle and L. Vaidman, Phys. Rev. A 37, 4052 (1988). 